\title{
GMR
}

\section{Identification of significant pathway cross-talk in rheumatoid arthritis by the Monte Carlo cross-validation method}

\author{
W. Song ${ }^{1}$, Y.M. Zhang ${ }^{1}$, T. Ma ${ }^{1}$, J. Wang ${ }^{1}$ and K.Z. Wang ${ }^{2}$ \\ ${ }^{1}$ Department of Joint Surgery, Xi'an Honghui Hospital, Xi'an, \\ Shanxi Province, China \\ ${ }^{2}$ Department of Orthopedics, The Second Affiliated Hospital of Xi' an Jiaotong \\ University (Xibei Hospital), Xi'an, Shanxi Province, China \\ Corresponding author: K.Z. Wang \\ E-mail: wangkunzhengw@yeah.net \\ Genet. Mol. Res. 16 (2): gmr16029142 \\ Received September 2, 2016 \\ Accepted February 17, 2017 \\ Published Abril 5, 2017 \\ DOI http://dx.doi.org/10.4238/gmr16029142 \\ Copyright $(2017$ The Authors. This is an open-access article distributed under the terms of \\ the Creative Commons Attribution ShareAlike (CC BY-SA) 4.0 License.
}

\begin{abstract}
We attempted to identify significant pathway crosstalk in rheumatoid arthritis (RA) by the Monte Carlo cross-validation (MCCV) method. We therefore obtained and preprocessed the gene expression profile of RA. MCCV involves identifying differentially expressed genes (DEGs), identifying differential pathways (DPs), calculating the discriminating score (DS) of the pathway cross-talk, and random forest (RF) classification. We carried out 50 bootstrap iterations of MCCV to identify the key instances of pathway cross-talk involved in RA. We identified a total of 17 significant DEGs and 15 significant DPs by comparing RA samples and normal controls. We found the most significant difference between RA and the normal controls in the eIF4 and $\mathrm{p} 70 \mathrm{~S} 6 \mathrm{~K}$ signaling regulation pathway. Furthermore, we identified 10 instances of pathway cross-talk with the best classification performance for RA and normal controls, using the RF classification model. All of the top 10 pathway pairs involved cross-talk with eIF4 and p70S6K signaling regulation, and the other 10 pathways were immune-related.
\end{abstract}

Genetics and Molecular Research 16 (2): gmr16029142 
By MCCV, we identified one critical DP and 10 significant instances of pathway cross-talk in RA. We propose that the eIF4 and p70S6K signaling regulation pathway and the other significant instances of pathway cross-talk play key roles in the occurrence and development of RA, and are potential predictive and prognostic markers for RA.

Key words: Rheumatoid arthritis; Monte Carlo cross-validation; Pathway cross-talk; eIF4 and p70S6K signaling regulation

\section{INTRODUCTION}

Rheumatoid arthritis (RA) is a common, chronic, inflammatory, and destructive arthropathy that cannot be cured; it involves substantial personal, social, and economic costs (McInnes and Schett, 2011). Current studies indicate that genetic and environmental factors, such as smoking and infection, contribute to the development of RA (Liu et al., 2013; Hensvold et al., 2015). However, its exact cause remains unclear.

Pathway analysis is a very effective means of gaining insight into the mechanisms underlying diseases (Goeman and Bühlmann, 2007). T cells, B cells, and the orchestrated interaction of pro-inflammatory cytokines play key roles in the pathophysiology of RA (Choy, 2012). Cytokine-related signaling pathways are also associated with the disease (McInnes and Schett, 2007; Bartok and Firestein, 2010). Most currently available methods consider such pathways to be independent mechanisms (Yi et al., 2013), and do not take into account the correlation between pathways, which is referred to as cross-talk (Liu et al., 2010). However, pathways are likely to interact with or influence each other in biological processes ( $\mathrm{Li}$ et al., 2008). Therefore, identifying pathway interactions through genetic screening is an effective biological approach to uncovering pathway cross-talk (Tong et al., 2004). There are several approaches to leave-one-out cross-talk validation, such as the $C_{p}$ statistics method (Mallows, 1973), the jackknife and bootstrap method (Efron, 1986), and cross validation (CV) (Wold, 1978). These methods are good for calibration but poor for prediction. Monte Carlo crossvalidation $(\mathrm{MCCV})$, an asymptotically consistent method for determining the number of components in a calibration model, avoids an unnecessarily large model and therefore reduces the risk of over-fitting model calibration (Xu and Liang, 2001).

We performed MCCV to carry out pathway cross-talk analysis on the gene expression profile of RA to gain a better understanding of its etiology and underlying mechanism. To achieve this, we first obtained and preprocessed the gene expression profile of RA. We then implemented MCCV to identify the key instances of pathway cross-talk involved in RA. This comprised four steps: identifying differentially expressed gene (DEGs); identifying differential pathways (DPs); calculating the discriminating score (DS) of the pathway cross-talk; and random forest $(\mathrm{RF})$ classification. Our results might provide new insight into the molecular pathology and underlying genetic targets of RA.

\section{MATERIAL AND METHODS}

\section{Data recruitment and preprocessing}

The gene expression profile of RA (accession number E-GEOD-45291) (Bienkowska

Genetics and Molecular Research 16 (2): gmr16029142 
et al., 2014) was downloaded from the ArrayExpress database (http://www.ebi.ac.uk/ arrayexpress/). It exists on the A-GEOD-13158 - [HT_HG-U133_Plus_PM] Affymetrix HT HG-U133+ PM Array Plate platform. This dataset comprised a total of 805 whole blood samples, including those from: 378 RA patients who had responded satisfactorily to a diseasemodifying anti-rheumatic drug; 115 RA patients who had not responded satisfactorily to tumor necrosis factor (TNF)-blocking therapy, and had discontinued TNF blocking treatment for at least 90 days; 292 patients with systemic lupus erythematosus; and 20 healthy volunteer donors. We selected 115 RA patients who had not responded satisfactorily to TNF-blocking therapy and 20 normal controls for the study. Importantly, the 115 RA patients had discontinued TNFblocking treatment for at least 90 days, so the effects of TNF on the blood transcriptome of the RA patients were considered to have been eliminated. We then downloaded the microarray data and annotation files. The gene expression profile at the probe level was converted to gene symbols. Finally, we wiped off the duplicated symbols, leaving a total of 20,276 gene symbols.

\section{MCCV}

In the present study, we implemented 50 bootstrap iterations of MCCV to identify the instances of pathway cross-talk associated with RA, and quantify the cross-talk. Figure 1 is a flowchart of the analytical procedure. In each bootstrap iteration, the expression profile data were divided randomly into two groups in a $6: 4$ ratio, of which $60 \%$ was treated as a training set and the remaining $40 \%$ was treated as a testing set. Cross-validation between the training and testing sets was carried out in each bootstrap iteration. Thus, MCCV randomly produced new training and testing sets in each bootstrap iteration, which comprised the following four steps: identifying DEGs, identifying differential pathways, calculating the DS value of the DP cross-talk, and RF classification.

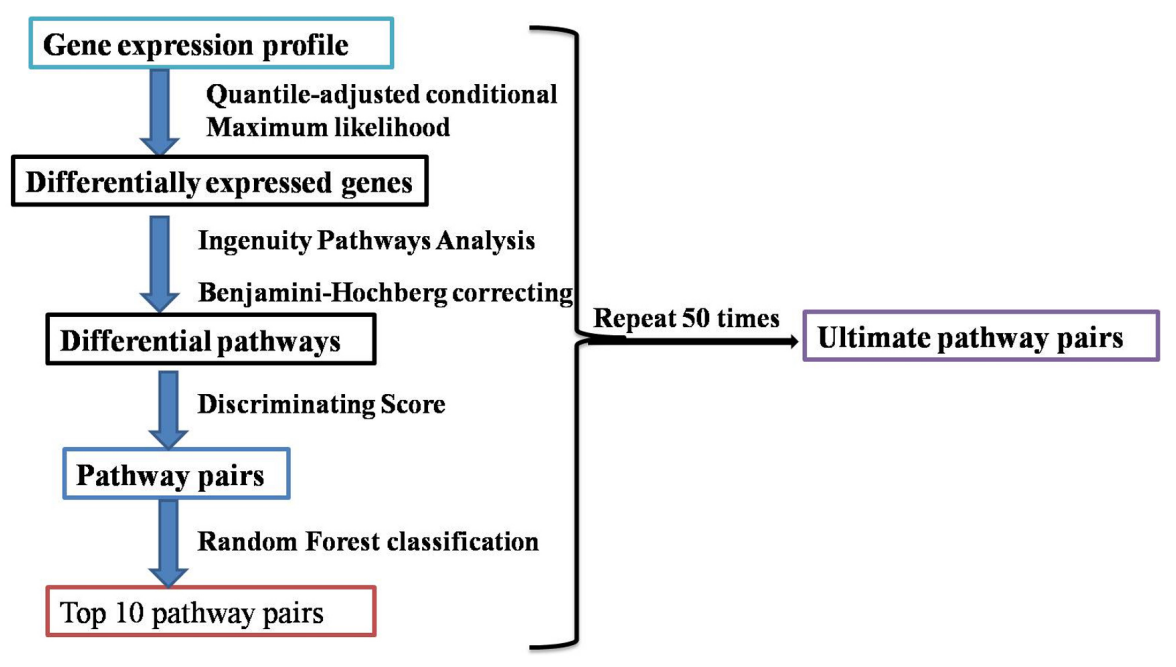

\section{Monte Carlo cross-Validation}

Figure 1. Schematic description of the method of Monte Carlo Cross-Validation (MCCV) that used in the research.

Genetics and Molecular Research 16 (2): gmr16029142 


\section{Identifying DEGs}

Before identifying DEGs, we carried out data preprocessing to further control the quality of the data and eliminate batch effects caused by experimental parameters and other factors. Quantiles (Bolstad et al., 2003) were applied to standardize the normalization of the expression data. The genes whose quantile means across all samples were higher than 0.25 -fold were selected for further analysis. To calculate the differential expression levels, the quantileadjusted conditional maximum likelihood (qCML) function of the edgeR package (Robinson et al., 2010) was applied to identify the DEGs in the RA samples and normal controls. The false discovery rate (FDR) was determined following the Benjamini-Hochberg procedure for multiple testing correction (Benjamini and Hochberg, 1995). DEGs were defined as those in which the differences in expression between the RA samples and the normal controls were significant (i.e., had FDR-adjusted P-values of $<0.05$ ).

\section{Identifying DPs}

We performed a pathway analysis based on the DEGs to ascertain DEG-related functional relevance in RA. The original pathways were obtained from the Ingenuity Pathway Analysis (IPA) database (http://www.ingenuity.com), which is a web-based software application that enables researchers to analyze data derived from the expression of single nucleotide polymorphism (SNP) microarrays, RNA sequencing, proteomics, metabolomics experiments, and small-scale experiments [such as polymerase chain reactions (PCRs)] that generate gene or protein lists (Krämer et al., 2014). In the current study, a total of 589 pathways (covering 5169 genes) was retrieved from the IPA database. To highlight the regulatory apparatus driving phenotypic differentiation, and to identify the pathways responsible for coordinating their activity, the DEG-related pathways were retrieved on the basis of the IPA pathways. The Fisher's test was performed to evaluate the significance of the pathways. The $\mathrm{P}$ values were adjusted by FDR according to the Benjamini-Hochberg procedure for multiple testing correction (Benjamini and Hochberg, 1995). Pathways with FDR-adjusted P values < 0.05 were considered DPs.

\section{Calculating the DS for pathway cross-talk}

To further define the relationship between two pathways, a DS (Bertucci et al., 2004) was computed by comparing the gene expression levels of each pair of pathways. The DS was calculated according to Equation (1):

$$
D S=\frac{\left|\left(M_{a}-M_{b}\right)\right|}{S_{a}+S_{b}}
$$

Where $\mathrm{M}_{\mathrm{a}}$ and $\mathrm{S}_{\mathrm{a}}$ represent the mean and standard deviation of gene expression levels in pathway $a$, and $\mathrm{M}_{b}$ and $\mathrm{S}_{\mathrm{b}}$ represent the corresponding values in pathway $b$. The DS is a measure of pathway cross-talk, and a larger DS indicates a greater difference in activity between two pathways.

Genetics and Molecular Research 16 (2): gmr16029142 


\section{RF classification}

Over the past few years, RF has attracted the attention of researchers who carry out analyses of microarray classifications and other high-dimensional molecular data (Díaz-Uriarte and Alvarez de Andrés, 2006; Statnikov et al., 2008). RF is an algorithm for classification that was developed by Leo Breiman (2001) and uses an ensemble of classification trees (Hastie et al., 2009). Each of the classification trees is built using a bootstrap sample of the data, and the candidate set of variables is a random subset of the variables at each split (Díaz-Uriarte and Alvarez de Andrés, 2006). In the present study, we carried out a 10-fold cross-validation for RF classification of the samples according to the DS of the pathway cross-talk. The area under the receiver operating characteristics curve (AUC) was used to evaluate classification accuracy. The AUC values were ranked in descending order, and the top 10 instances of pathway cross-talk were identified.

We applied 50 bootstrap iterations of the process described above, which randomly generated new training and testing partitions each time. For each bootstrap iteration, DEGs, DPs, and DSs for pathway cross-talk were analyzed. Based on the training dataset, we detected the DSs for the top 10 instances of pathway cross-talk with the best AUC values. We then used the testing dataset to validate the top 10 instances of pathway cross-talk. After all 50 bootstrap iterations, the top 10 instances of pathway cross-talk were selected by ranking them according to their AUC values. We counted the times that an instance of pathway cross-talk appeared in the 50 bootstrap iterations. In the present study, the top 10 pathways across all 50 bootstrap iterations were regarded as significant instances of pathway cross-talk for RA.

\section{RESULTS}

\section{Identifying DEGs}

We carried out pathway cross-talk analysis of the gene expression profile of RA to gain a better understanding of the pathological mechanism underlying RA. The analysis comprised 50 bootstrap iterations of MCCV. After quantile normalization, we identified 15,207 genes with higher than 0.25 -fold quantile means across all samples. DEGs were identified by applying the qCML function of the edgeR package. We identified 17 significant DEGs with FDR-adjusted $\mathrm{P}$ values $<0.05$ (Table 1).

Table 1. Differentially expressed genes in rheumatoid arthritis via Monte Carlo Cross-Validation.

\begin{tabular}{l|c|c|l|c|c}
\hline Gene & Times & P value & Gene & Times & P value \\
\hline HLA-DQA1 & 48 & 0.001 & SBNO2 & 15 & 0.029 \\
\hline KDM5D & 49 & 0.001 & SLC39A4 & 15 & 0.030 \\
\hline RPS4Y1 & 49 & 0.005 & ZKSCAN5 & 1 & 0.030 \\
\hline EIF1AY & 49 & 0.008 & EFCAB4A & 1 & 0.036 \\
\hline CYorf15A & 21 & 0.014 & C8orf82 & 1 & 0.038 \\
\hline XIST & 11 & 0.014 & PEMT & 6 & 0.042 \\
\hline DFNB31 & 31 & 0.015 & HIST1H2AD & 1 & 0.043 \\
\hline CYorf15B & 28 & 0.016 & HLA-DRB4 & & 0.048 \\
\hline TSIX & 1 & 0.018 & & & \\
\hline
\end{tabular}

\section{Identifying differential pathways}

The biological pathways in the IPA database were downloaded as original pathways.

Genetics and Molecular Research 16 (2): gmr16029142 
There were 589 pathways (containing 5169 genes) in all. DPs under the threshold value of P $<0.05$ were identified in each bootstrap iteration of MCCV. After 50 bootstraps iterations, 15 DPs were obtained (Table 2). The eIF4 and p70S6K signaling regulation pathway showed the most significant difference $(\mathrm{P}=0.001)$.

Table 2. Differential pathways in rheumatoid arthritis via Monte Carlo Cross-Validation.

\begin{tabular}{|c|c|c|c|}
\hline Pathway & Gene size & Times & Pvalue \\
\hline Regulation of eIF4 and p70S6K Signaling & 142 & 49 & 0.001 \\
\hline Antigen Presentation Pathway & 34 & 48 & 0.012 \\
\hline Graft-versus-Host Disease Signaling & 39 & 48 & 0.012 \\
\hline T Helper Cell Differentiation & 62 & 48 & 0.022 \\
\hline IL-4 Signaling & 70 & 48 & 0.024 \\
\hline Altered T Cell and B Cell Signaling in Rheumatoid Arthritis & 76 & 48 & 0.025 \\
\hline VEGF Signaling & 89 & 48 & 0.03 \\
\hline CD28 Signaling in T Helper Cells & 107 & 37 & 0.031 \\
\hline iCOS-iCOSL Signaling in T Helper Cells & 97 & 44 & 0.031 \\
\hline PKC _ Signaling in T Lymphocytes & 107 & 37 & 0.031 \\
\hline Type I Diabetes Mellitus Signaling & 101 & 44 & 0.033 \\
\hline Dendritic Cell Maturation & 159 & 19 & 0.034 \\
\hline EIF2 Signaling & 171 & 20 & 0.035 \\
\hline Role of NFAT in Regulation of the Immune Response & 160 & 19 & 0.035 \\
\hline mTOR Signaling & 182 & 19 & 0.037 \\
\hline
\end{tabular}

\section{Identifying key instances of pathway cross-talk}

In this step, the RF classification model was used to measure the classification accuracy of instances of pathway cross-talk based on DSs. The DSs of all instances of pathway cross-talk were obtained via 50 bootstrap iterations. Next, 10-fold cross-validation was performed to carry out RF classification on the samples according to the DSs of the instances of pathway cross-talk, and the AUC values were used to evaluate the classification results. The top 10 instances of pathway cross-talk with the best classification performance for the RA samples and normal controls were defined by ranking all the AUC values in descending order after 50 bootstrap iterations. Details of pathway cross-talk between regulation of eIF4 and p70S6K signaling and the other 10 pathways are listed in Table 3. The role of eIF4 and p70S6K signaling regulation in RA cannot be ignored. The top 9 instances of pathway crosstalk showed the same classification performances, with AUC values of 0.998, and the pathway cross-talk of eIF4 and p70S6K signaling regulation and dendritic cell maturation had an AUC value of 0.981 .

Table 3. The top 10 pathway cross-talks according to the best classification accuracy.

\begin{tabular}{l|c|c}
\hline Pathway cross-talk & AUC & Frequency \\
\hline Regulation of eIF4 and p70S6K signaling; Graft-versus-host disease signaling & 0.998 & 16 \\
\hline Regulation of eIF4 and p70S6K signaling; Antigen presentation pathway & 0.998 & 16 \\
\hline Regulation of eIF4 and p70S6K signaling; T helper cell differentiation & 0.998 & \\
\hline Regulation of eIF4 and p70S6K signaling; IL-4 signaling & 0.998 & 15 \\
\hline Regulation of eIF4 and p70S6K signaling; Altered T cell and B cell signaling in rheumatoid arthritis & 0.998 & 17 \\
\hline Regulation of eIF4 and p70S6K signaling; iCOS-iCOSL signaling in Thelper cells & 0.998 & 14 \\
\hline Regulation of eIF4 and p70S6K signaling; Type I diabetes mellitus signaling & 0.998 & 11 \\
\hline Regulation of eIF4 and p70S6K signaling; CD28 signaling in T helper cells & 0.998 & 10 \\
\hline Regulation of eIF4 and p70S6K signaling; PKC signaling in T lymphocytes & 0.998 & 7 \\
\hline Regulation of eIF4 and p70S6K signaling; Dendritic cell maturation & 0.981 & 7 \\
\hline
\end{tabular}

AUC: the area under the receiver operating characteristics curve. 
To further define the importance of pathway cross-talk, we calculated the frequency of the top 10 individual instances of pathway cross-talk in 50 bootstrap iterations (Figure 2). The pathway cross-talk of eIF4 and p70S6K signaling regulation and IL-4 signaling was observed in 17 bootstrap iterations. Two instances of pathway cross-talk (regulation of eIF4 and p70S6K signaling and graft-versus-host disease signaling, and regulation of eIF4 and p70S6K signaling and antigen presentation pathway) were observed in 16 bootstrap iterations.

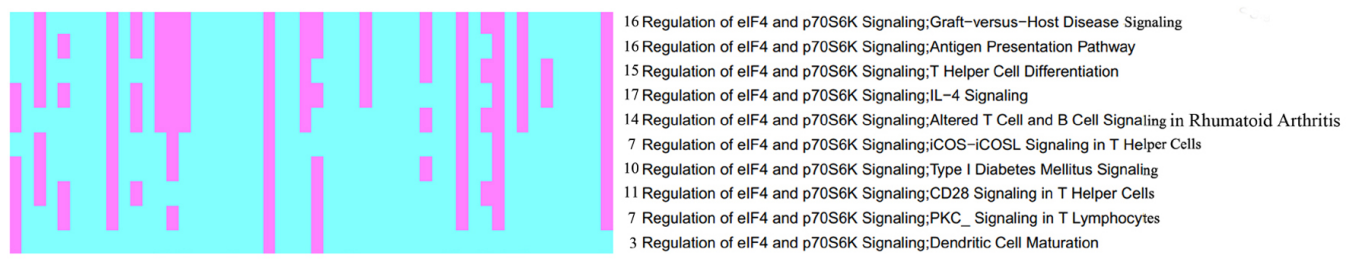

Figure 2. The heatmap of the key pathway cross-talks ranked according to the AUC values in descending order after repeating 50 bootstraps in the training dataset.

\section{Validation analysis}

We used another independent RA dataset (E-GEOD-15573) to validate the results by MCCV. E-GEOD-15573 (Teixeira et al., 2009) comprised 33 peripheral blood samples from $18 \mathrm{RA}$ patients and 15 normal controls. The analysis procedure was the same as that described above. We screened the top 10 instances of pathway cross-talk by MCCV analysis of dataset E-GEOD-15573. Among them, three instances of pathway cross-talk were validated, including regulation of eIF4 and p70S6K signaling-antigen presentation, regulation of eIF4 and p70S6K signaling-altered $\mathrm{T}$ cell and B cell signaling in rheumatoid arthritis, and regulation of eIF4 and p70S6K signaling-IL-4 signaling. Furthermore, two other instances of pathway cross-talk related to regulation of eIF4 and p70S6K signaling were identified. The results indicate the critical roles of eIF4 and p70S6K signaling regulation in RA, and show the feasibility and repeatability of MCCV analysis in RA studies.

\section{DISCUSSION}

RA is a chronic, progressive, inflammatory autoimmune disease that is mainly caused by articular, extra-articular, and systemic effects (Okada et al., 2014). During the past few years, efforts have been made to gain a better understanding of RA. However, the etiology and underlying mechanism of RA have not been fully elucidated. Therefore, in the present study, we performed MCCV to conduct pathway cross-talk analysis based on the gene expression profile of RA. We found that the eIF4 and p70S6K signaling regulation pathway was most relevant to RA, and the top 10 instances of pathway cross-talk were all related to the eIF4 and p70S6K signaling regulation pathway. To further understand the relationships between these pathways, we have included an in-depth discussion, as follows.

The present study suggests that the eIF4 and p70S6K signaling regulation pathway plays an important role in RA. Lin et al. (2017) indicated that regulation of the eIF4 and p70S6K signaling pathway is involved in polyarticular juvenile idiopathic arthritis. Both eIF4 (eukaryotic initiation factor-4) and p70S6K (p70 ribosomal S6 kinase) play critical

Genetics and Molecular Research 16 (2): gmr16029142 
roles in translational regulation. Researchers have indicated that $\mathrm{p} 70 \mathrm{~S} 6 \mathrm{~K}$ and the binding protein eukaryotic translation initiation factor eIF4 are two main phosphorylation targets of mammalian target of rapamycin (mTOR) (Young and Nickerson-Nutter, 2005). The pathway of mTOR complex 1 is critical for the initiation of inflammatory reactions and cell homeostasis in human RA synovial cells (Yin et al., 2015). Moreover, mTOR signaling is active in the synovial membrane of patients with RA, particularly in synovial osteoclasts (Cejka et al., 2010). In this study, mTOR was identified as a differential pathway in RA.

RA is a well-known inflammatory autoimmune disease. However, we unexpectedly discovered that all of the top 10 instances of pathway cross-talk, such as IL-4 signaling, were related to immune response. Interleukins (ILs) are a group of cytokines (secreted proteins and signal molecules) that are expressed first by white blood cells (leukocytes) (Brocker et al., 2010). In RA, articular inflammation seems to be caused by the expression of inflammatory cytokines and chemokines that determine the activation and proliferation of the synovial lining, and by inflammatory cell recruitment and B cell activation with autoantibody production (Samson et al., 2012). The IL-1 family of cytokines (IL-1a, IL-1b, IL-18, and IL-33) are highly expressed in RA (Bessis et al., 2000; Li et al., 2016). Furthermore, IFN-b, TGFb, IL-4, and IL-13 increase the expression of IL-1Ra while diminishing the production of IL-1 (Chizzolini et al., 2009). Moreover, a previous report analyzing the blood mononuclear cells obtained from RA patients indicates that the apoptotic-dependent reduction of Th1/Th17/Treg cells is accompanied by the enhanced expression of IL-4 (Guggino et al., 2014).

Therefore, we conclude that the MCCV method for identifying significant pathway cross-talk in RA is effective. By this method, we successfully identified 10 significant instances of pathway cross-talk, all of which were related to the regulation of eIF4 and p70S6K signaling. We propose that these instances of pathway cross-talk play key roles in the occurrence and development of RA, and are potential predictive and prognostic markers for RA.

\section{Conflicts of interest}

The authors declare no conflict of interest.

\section{ACKNOWLEDGMENTS}

Research conducted under the careful supervision and with the strong support of Professor Kunzheng Wang. We would like to thank the members of the research group at the Department of Joint Surgery, Xi' an Honghui Hospital, for all their help. We are also grateful to the Beijing Springer Medical Research Institute for professional translation and for improving the paper.

\section{REFERENCES}

Bartok B and Firestein GS (2010). Fibroblast-like synoviocytes: key effector cells in rheumatoid arthritis. Immunol. Rev. 233: 233-255. http://dx.doi.org/10.1111/j.0105-2896.2009.00859.x

Benjamini Y and Hochberg Y (1995). Controlling the false discovery rate: a practical and powerful approach to multiple testing. J. The Royal Statist. Society. Series B (Methodological) 57: 289-300.

Bertucci F, Finetti P, Rougemont J, Charafe-Jauffret E, et al. (2004). Gene expression profiling for molecular characterization of inflammatory breast cancer and prediction of response to chemotherapy. Cancer Res. 64: 8558-8565. http://dx.doi. org/10.1158/0008-5472.CAN-04-2696

Genetics and Molecular Research 16 (2): gmr16029142 
Bessis N, Guéry L, Mantovani A, Vecchi A, et al. (2000). The type II decoy receptor of IL-1 inhibits murine collageninduced arthritis. Eur. J. Immunol. 30: 867-875. http://dx.doi.org/10.1002/1521-4141(200003)30:3<867::AIDIMMU867>3.0.CO;2-M

Bienkowska J, Allaire N, Thai A, Goyal J, et al. (2014). Lymphotoxin-LIGHT pathway regulates the interferon signature in rheumatoid arthritis. PLoS One 9: e112545. http://dx.doi.org/10.1371/journal.pone.0112545

Bolstad BM, Irizarry RA, Astrand M and Speed TP (2003). A comparison of normalization methods for high density oligonucleotide array data based on variance and bias. Bioinformatics 19: 185-193. http://dx.doi.org/10.1093/ bioinformatics/19.2.185

Breiman L (2001). Random forests. Mach. Learn. 45: 5-32. http://dx.doi.org/10.1023/A:1010933404324

Brocker C, Thompson D, Matsumoto A, Nebert DW, et al. (2010). Evolutionary divergence and functions of the human interleukin (IL) gene family. Hum. Genomics 5: 30-55. http://dx.doi.org/10.1186/1479-7364-5-1-30

Cejka D, Hayer S, Niederreiter B, Sieghart W, et al. (2010). Mammalian target of rapamycin signaling is crucial for joint destruction in experimental arthritis and is activated in osteoclasts from patients with rheumatoid arthritis. Arthritis Rheum. 62: 2294-2302. http://dx.doi.org/10.1002/art.27504

Chizzolini C, Dayer JM and Miossec P (2009). Cytokines in chronic rheumatic diseases: is everything lack of homeostatic balance? Arthritis Res. Ther. 11: 246 http://dx.doi.org/10.1186/ar2767.

Choy E (2012). Understanding the dynamics: pathways involved in the pathogenesis of rheumatoid arthritis. Rheumatology (Oxford) 51 (Suppl 5): v3-v11. http://dx.doi.org/10.1093/rheumatology/kes113

Díaz-Uriarte R and Alvarez de Andrés S (2006). Gene selection and classification of microarray data using random forest. BMC Bioinformatics 7: 3. http://dx.doi.org/10.1186/1471-2105-7-3

Efron B (1986). How biased is the apparent error rate of a prediction rule? J. Am. Stat. Assoc. 81: 461-470. http://dx.doi. org/10.1080/01621459.1986.10478291

Goeman JJ and Bühlmann P (2007). Analyzing gene expression data in terms of gene sets: methodological issues. Bioinformatics 23: 980-987. http://dx.doi.org/10.1093/bioinformatics/btm051

Guggino G, Giardina AR, Raimondo S, Giardina G, et al. (2014). Targeting IL-6 signalling in early rheumatoid arthritis is followed by Th1 and Th17 suppression and Th2 expansion. Clin. Exp. Rheumatol. 32: 77-81.

Hastie T, Tibshirani R and Friedman J (2009). The elements of statistical learning: Data mining, inference, and prediction. 2nd edition. Springer-Verlag, New York.

Hensvold AH, Magnusson PK, Joshua V, Hansson M, et al. (2015). Environmental and genetic factors in the development of anticitrullinated protein antibodies (ACPAs) and ACPA-positive rheumatoid arthritis: an epidemiological investigation in twins. Ann. Rheum. Dis. 74: 375-380. http://dx.doi.org/10.1136/annrheumdis-2013-203947

Krämer A, Green J, Pollard J, Jr. and Tugendreich S (2014). Causal analysis approaches in Ingenuity Pathway Analysis. Bioinformatics 30: 523-530. http://dx.doi.org/10.1093/bioinformatics/btt703

Li LL, Deng XF, Li JP, Ning N, et al. (2016). Association of IL-18 polymorphisms with rheumatoid arthritis: a metaanalysis. Genet. Mol. Res. 15: http://dx.doi.org/10.4238/gmr.15017389.

Li Y, Agarwal P and Rajagopalan D (2008). A global pathway crosstalk network. Bioinformatics 24: 1442-1447. http:// dx.doi.org/10.1093/bioinformatics/btn200

Lin S, Wang Y, Mu S, Zhang J, et al. (2017). Pathway analysis based on Monte Carlo Cross-Validation in polyarticular juvenile idiopathic arthritis. Pathol. Res. Pract. 213: 7-12. http://dx.doi.org/10.1016/j.prp.2016.04.010

Liu Y, Aryee MJ, Padyukov L, Fallin MD, et al. (2013). Epigenome-wide association data implicate DNA methylation as an intermediary of genetic risk in rheumatoid arthritis. Nat. Biotechnol. 31: 142-147. http://dx.doi.org/10.1038/nbt.2487

Liu ZP, Wang Y, Zhang XS and Chen L (2010). Identifying dysfunctional crosstalk of pathways in various regions of Alzheimer's disease brains. BMC Syst. Biol. 4 (Suppl 2): S11 http://dx.doi.org/10.1186/1752-0509-4-S2-S11.

Mallows CL (1973). Some comments on C P. Technometrics 15: 661-675.

McInnes IB and Schett G (2007). Cytokines in the pathogenesis of rheumatoid arthritis. Nat. Rev. Immunol. 7: 429-442. http://dx.doi.org/10.1038/nri2094

McInnes IB and Schett G (2011). The pathogenesis of rheumatoid arthritis. N. Engl. J. Med. 365: 2205-2219. http://dx.doi. org/10.1056/NEJMra1004965

Okada Y, Wu D, Trynka G, Raj T, et al.; RACI consortium; GARNET consortium (2014). Genetics of rheumatoid arthritis contributes to biology and drug discovery. Nature 506: 376-381. http://dx.doi.org/10.1038/nature12873

Robinson MD, McCarthy DJ and Smyth GK (2010). edgeR: a Bioconductor package for differential expression analysis of digital gene expression data. Bioinformatics 26: 139-140. http://dx.doi.org/10.1093/bioinformatics/btp616

Samson M, Audia S, Janikashvili N, Ciudad M, et al. (2012). Brief report: inhibition of interleukin-6 function corrects Th17/Treg cell imbalance in patients with rheumatoid arthritis. Arthritis Rheum. 64: 2499-2503. http://dx.doi. org/10.1002/art.34477

Genetics and Molecular Research 16 (2): gmr16029142 
Statnikov A, Wang L and Aliferis CF (2008). A comprehensive comparison of random forests and support vector machines for microarray-based cancer classification. BMC Bioinformatics 9: $319 \mathrm{http} / / / \mathrm{dx}$. doi.org/10.1186/1471-2105-9-319.

Teixeira VH, Olaso R, Martin-Magniette ML, Lasbleiz S, et al. (2009). Transcriptome analysis describing new immunity and defense genes in peripheral blood mononuclear cells of rheumatoid arthritis patients. PLoS One 4: e6803. http:// dx.doi.org/10.1371/journal.pone. 0006803

Tong AHY, Lesage G, Bader GD, Ding H, et al. (2004). Global mapping of the yeast genetic interaction network. Science 303: 808-813. http://dx.doi.org/10.1126/science.1091317

Wold S (1978). Cross-validatory estimation of the number of components in factor and principal components models. Technometrics 20: 397-405. http://dx.doi.org/10.1080/00401706.1978.10489693

Xu Q-S and Liang Y-Z (2001). Monte Carlo cross validation. Chemom. Intell. Lab. Syst. 56: 1-11. http://dx.doi.org/10.1016/ S0169-7439(00)00122-2

Yi CQ, Ma CH, Xie ZP, Cao Y, et al. (2013). Comparative genome-wide gene expression analysis of rheumatoid arthritis and osteoarthritis. Genet. Mol. Res. 12: 3136-3145. http://dx.doi.org/10.4238/2013.March.11.3

Yin G, Li Y, Yang M, Cen XM, et al. (2015). Pim-2/mTORC1 pathway shapes inflammatory capacity in rheumatoid arthritis synovial cells exposed to lipid peroxidations. BioMed Res. Int. 2015: $240210 \mathrm{http} / / \mathrm{dx}$.doi.org/10.1155/2015/240210.

Young DA and Nickerson-Nutter CL (2005). mTOR--beyond transplantation. Curr. Opin. Pharmacol. 5: 418-423. http:// dx.doi.org/10.1016/j.coph.2005.03.004

Genetics and Molecular Research 16 (2): gmr16029142 